\title{
Advances in tailoring the porosity of tannin-based carbon xerogels
}

Natalia Rey-Raap ${ }^{1}$, Andrzej Szczurek ${ }^{2}$, Vanessa Fierro ${ }^{2}$, Alain Celzard ${ }^{2}$, J. Angel

Menéndez ${ }^{1}$, Ana Arenillas ${ }^{1 *}$

${ }^{1}$ Instituto Nacional del Carbón, CSIC, Apdo 73, 33080 Oviedo, España

${ }^{2}$ Institut Jean Lamour -UMR CNRS-Université de Lorraine n7198, ENSTIB, 27 rue Philippe Séguin, CS 60036, 88026 Épinal Cedex, France

(1)

\section{ABSTRACT}

Usually, carbon xerogels are obtained from resorcinol-formaldehyde organic gels. However, more cost-effective and eco-friendly carbon xerogels can be synthesized by using tannins instead of resorcinol, provided that a suitable surfactant is added to prevent the collapse of the structure. The use of tannin, a natural phenolic compound derived from wood, allows obtaining carbon xerogels with controlled porosity, as the porous properties of these materials can be tailored by an appropriate choice of the synthesis conditions. In this work, tannin-formaldehyde xerogels containing different amounts of surfactant and formaldehyde were synthesized in order to evaluate their effect on the porous structure and chemical composition. It was found that porosity and density depend greatly on the amount of surfactant. The lowest density and highest porosity values $-0.34 \mathrm{~g} / \mathrm{cm}^{3}$ and $78 \%$, respectively- were obtained by adding $10 \mathrm{wt} . \%$ of surfactant. It was also found that S-doped carbon xerogels can be easily synthesized due to the strong affinity between the carbon in the structure and the sulphur from the surfactant. Furthermore, statistical analysis showed that there is interdependence between the effect of formaldehyde and the surfactant, especially in the case of volume and pore size. Hence, the choice of the appropriate surfactant-formaldehyde 
concentration is essential for controlling the formation of the porous polymeric structure.

28 Keywords: carbon gel, tannin, surfactant, controlled porosity

\section{$30 \quad 1$ Introduction}

31 Carbon gels are porous materials that are obtained by drying and carbonizing organic polymer-based gels (Elkhatat and Al-Muhtaseb, 2011). The great advantage of these materials is that, depending on the preparation method and the nature, concentration and chemical composition of the precursors, the porous structure can be controlled (Matos et al., 2006; Rey-Raap et al., 2014). Hence, their final properties can be tailored to fit the requirement of a specific application. The versatility of carbon xerogels is the reason why these materials are perfect candidates for various applications such as adsorption (Job et al., 2005), hydrogen storage (Zubizarreta et al., 2009), electrode material for supercapacitors or batteries (Braghiroli et al., 2015b; Roberts et al., 2014), catalyst support (Yang et al., 2014), thermal insulators (Tannert et al., 2015), etc.

The most common method of synthesizing organic gels is by means of the polymerization of resorcinol with formaldehyde in the presence of a solvent (Elkhatat and Al-Muhtaseb, 2011). The organic gel obtained can be dried under supercritical conditions, by freeze-drying process or by evaporation, which give rise to aerogels, cryogels and xerogels, respectively (Rey-Raap et al., 2014). The preparation of aerogels and cryogels involves cumbersome solvent exchange processes and complex drying stages (Al-Muhtaseb and Ritter, 2003). Thus, evaporative drying is the quickest and

49 simplest method and therefore, yields the most cost-competitive materials. 
51 Although resorcinol and formaldehyde are the most commonly employed reagents, they are costly and harmful to the environment. Consequently, studies with less expensive and greener precursors have been undertaken over the last few decades (Rey-Raap et al., 2015; Szczurek et al., 2014). All the hydroxylated benzenes tested to date contain reactive hydroxyl groups which make them similar to resorcinol in the way they react with aldehydes (Arbenz and Averous, 2014; Lochab et al., 2014). Of all the possible reagents, the use of tannin, a natural counterpart to resorcinol, for the synthesis of carbon gels has attracted a great deal of interest in recent years due to the characteristics of this reagent, which is "eco-friendly", cheap and non-toxic (Braghiroli et al., 2015a;

\section{García et al., 2014).}

As in the synthesis of resorcinol-formaldehyde carbon xerogels, the concentrations of all the reagents employed to polymerize tannin with formaldehyde (i.e., tannin,

64 formaldehyde, solvent and catalyst), play an important role in tailoring the final 65 properties of these porous materials (Amaral-Labat et al., 2013b; Rey-Raap et al., 66 2014). These concentrations have been reported in the literature to be related to the $\mathrm{pH}$, the percentage of solids and the weight ratio of tannin to formaldehyde (Amaral-Labat et al., 2013b; Szczurek et al., 2014). The $\mathrm{pH}$ has been one of the most studied variables as it has a great influence on the final properties of tannin-formaldehyde carbon gels over the entire range of porosity (Amaral-Labat et al., 2013a; Amaral-Labat et al., 2015; Amaral-Labat et al., 2013b; Szczurek et al., 2011a; Szczurek et al., 2011b). Other studies focused on the influence of the $\mathrm{pH}$ and percentage of solids, have reported that a decrease in $\mathrm{pH}$ and percentage of solids leads to an increase in macroporosity, while mesoporosity reaches its maximum value with an increase in the percentage of solids 
75 (Amaral-Labat et al., 2013b). It should be pointed out that most of the studies reported 76 to date were performed on aerogels and cryogels (Amaral-Labat et al., 2013b; Szczurek

77 et al., 2011a; Szczurek et al., 2011b), since the synthesis of carbon xerogels with tannin as precursor can only be achieved if surfactants are added (Amaral-Labat et al., 2013a; Amaral-Labat et al., 2015). In a study on the synthesis of tannin-based xerogels,

80 Amaral-Labat et al. reported the effect that modifying the concentration of Pluronic F81127 from 5 to $20 \mathrm{wt} \%$ has on the final porous properties (Amaral-Labat et al., 2015).

82 These authors found that xerogels turned from macroporous to mesoporous materials as

83 the amount of surfactant was increased. However, triblock copolymer surfactants such

84 as Pluronic require both an increase in temperature and the addition of an organic 85 solvent to dissolve and, hence, an extra step is required in the synthesis process 86 (Amaral-Labat et al., 2013a; Amaral-Labat et al., 2015). However, a recently published work has demonstrated that tannin-based xerogels can be synthesized by using sodium dodecyl sulphate (Rey-Raap et al., 2015). The use of this latter surfactant is expected to 89 give rise to S-doped materials. The introduction of heteroatoms during the synthesis 90 process is of great importance. For instance, the production of S-doped carbon materials

91 can enhance the properties of the started carbons and improve its suitability in some applications concerning sorption and energy storage/conversion devices (Kiciński et al., 93 2014).

94

95 Despite the importance of the concentration of formaldehyde for tailoring the porous 96 properties, the effect of modifying the tannin to formaldehyde weight ratio from 0.5 to 970.6 has been evaluated, to the best of our knowledge, in only one study (Szczurek et al., 2011a), that focuses on carbon cryogels. Yet it is essential to appreciate the importance 
99 of the concentration of formaldehyde for the polymerization reaction in order to control

100 porous properties of carbon xerogels.

101

102 The aim of the present work is to move a step closer towards the synthesis of tailored 103 porous carbon xerogels by a more cost-effective and environment-friendly route. To 104 achieve this goal, tannin-formaldehyde carbon xerogels were synthesized using different 105 amounts of formaldehyde and surfactant. The surfactant employed in the present study 106 does not require the addition of any organic solvent as it can be easily dissolved in 107 water. All the carbon xerogels synthesized were characterized from the point of view of 108 their chemical and porous structure in order to determine the effect of formaldehyde and 109 surfactant on the final porous properties of the xerogels.

\section{2. Experimental}

\section{$112 \quad 2.1$ Raw materials}

113 Commercial wattle tannin extracted from Acacia Mearnsii (De Wild) bark was used for

114 the present study. This raw material was kindly supplied by SilvaChimica (San Michele

115 Mondovi, Italy) under the name of Fintan OP. The industrial extraction process of 116 tannins has been explained in detail elsewhere (Braghiroli et al., 2014). Briefly, fresh

117 bark was subjected to leaching in a sodium bisulphite aqueous solution at $70^{\circ} \mathrm{C}$. The

118 resulting solutions were concentrated and then spray-dried to yield a light-brown 119 powder containing $80-82$ wt. \% of phenolic flavonoid materials, 4-6 wt. \% of water, 1

120 wt. \% of amino acids and other components such as monomeric and oligomeric 121 carbohydrates consisting of broken pieces of hemicellulose. The light-brown powder 122 thus obtained is referred to as tannin (T). 
124 Formaldehyde (37 wt. \% in water, stabilized by 10-15 wt. \% of methanol), was supplied

125 by Roth. The sodium dodecyl sulfate and sodium hydroxide were purchased from

126 Sigma Aldrich and from Carlo Erba, respectively.

127

$128 \quad 2.2$ Preparation of the carbon xerogels

129 Organic gels were synthesized by the polycondensation of tannin $(\mathrm{T})$ and formaldehyde

130 (F) using deionized water as solvent, sodium hydroxide as catalyst, and sodium dodecyl

131 sulfate (SDS) as surfactant (all the relevant data related to the reagents employed in this

132 study can be found in the Supporting Information). Tannin was first dissolved in

133 deionized water in open glass beakers under magnetic stirring at room temperature.

134 Once the tannin had dissolved, formaldehyde was introduced into the solution. Finally,

135 sodium dodecyl sulfate was added and the resulting mixture was stirred until a

136 homogeneous brown solution was obtained. All the gels were synthesized from $20 \mathrm{~mL}$

137 of precursor solution with a $\mathrm{pH}$ value fixed at 5.5 by adding sodium hydroxide. The

138 tannin/formaldehyde weight ratio varied between 0.6 and 2.6 whereas the percentage of

139 solids remained fixed at 25 wt. \% for all the samples. The concentration of sodium

140 dodecyl sulfate varied between 0 and $20 \mathrm{wt} . \%$.

141

142 Each precursor solution was poured into a sealed glass tube, with an inner diameter

143 value of $1.4 \mathrm{~cm}$, which was then placed in a ventilated oven at $85^{\circ} \mathrm{C}$ for $72 \mathrm{~h}$ to undergo

144 gelation and curing. Afterwards, the tube was opened and introduced again into the

145 oven at $85^{\circ} \mathrm{C}$ for $48 \mathrm{~h}$ for the organic gel to completely dry by evaporation of the

146 solvent. Once dry, each xerogel was carbonized at $900^{\circ} \mathrm{C}$ under nitrogen in a horizontal

147 tubular furnace. The residence time was $2 \mathrm{~h}$ and the heating rate was set at $5^{\circ} \mathrm{C} / \mathrm{min}$. The 
148 resultant carbon xerogels were labelled ' $\mathrm{TF}$ ' followed by the tannin-formaldehyde

149 weight ratio and the percentage of surfactant added, e.g., TF-1.2-10\%.

\subsection{Sample characterization}

152 The porous properties of the carbon xerogels were characterized by means of mercury

153 porosimetry (Autopore IV, Micromeritics). Measurements were performed between

1540.0035 and $414 \mathrm{MPa}$, and Washburn's equation was applied to the corresponding data

155 to obtain the specific pore volume and average pore size (see supporting information for

156 more details). Skeletal and bulk densities were determined by helium (Accupyc II 1340,

157 Micromeritics) and envelope (Geopyc 1360 Envelope Density Analyzer, Micromeritics)

158 pycnometry, respectively. The overall porosity was determined from the bulk and

159 skeletal densities. The morphology of the carbon structure was observed using a Quanta

160 FEG 600 scanning electron microscope and the chemical structure was investigated

161 using an Infrared Affinity-1 spectrometer (Shimadzu, Japan) by applying an average of

16220 scans at a maximum resolution of $4 \mathrm{~cm}^{-1}$ over a range of $4000-400 \mathrm{~cm}^{-1}$. For this

163 purpose, pellets were prepared by pressing $1 \mathrm{mg}$ of sample ground together with $100 \mathrm{mg}$

164 of dry potassium bromide. The chemical composition of the samples was determined by

165 elemental analysis (C, H, O, N, S), using a Vario EL Cube microanalyzer (Elementar).

\subsection{Statistical analysis}

168 Response surface methodology was applied to the porous characterization data in order

169 to assess the significance of each of the variables studied (amount of anionic surfactant 170 (SDS) and tannin/formaldehyde (T/F) weight ratio) and of any possible synergy

171 between them. Significance was determined by applying an analysis of variance

172 (ANOVA) to each of the four porous properties selected as responses: porosity, bulk 
173 density, pore volume and average pore size. The implementation of ANOVA allows the interaction between variables and their effect on the main porous properties to be evaluated with a minimum number of experiments. If the p-values obtained from the ANOVA were smaller than 0.05 , the corresponding variable would be assumed to have a significant effect on the response. An optimal design covering all the possible SDS-

$178 \mathrm{~T} / \mathrm{F}$ combinations together with repeated experimental runs were employed to obtain a 179 statistical model that adequately fitted the experimental results. The design matrix was generated by using a Design-Expert 9.0 Trial version from Stat-Ease Inc.

\section{$182 \quad 3$ Results and discussion}

\section{$183 \quad 3.1$ Statistical analysis}

184 Response surface methodology was applied to the four variables selected as responses: porosity, bulk density, pore volume and average pore size. Porosity and bulk density were adjusted to a quadratic function, whereas pore volume and average pore size were

187 fitted to a cubic function. The R-squared values for porosity, bulk density, pore volume 188 and average pore size were $0.90,0.93,0.89$ and 0.96 , respectively, indicating that the 189 calculated results are in good agreement with those obtained experimentally. Moreover, 190 the data obtained by employing a least square technique indicate that the model applied 191 to each response is significant (the ANOVA data for each response variable are shown 192 in Tables S1, S2, S3 and S4 in the Supporting Information). The linear and quadratic

193 terms for the amount of surfactant have a significant effect on porosity and density,

194 whereas the volume and the average size of the pores depend on linear, quadratic and 195 cubic terms of the amount of surfactant, and also on the $\mathrm{T} / \mathrm{F}$ weight ratio and the 196 interaction between the SDS and T/F weight ratio. Figure 1 shows the three-dimensional 197 surface plots constructed on the basis of model equations that represent the response 
198 surface curves of the independent variables (T/F weight ratio and percentage of

199 surfactant) versus the dependent variables: porosity (Figure 1a), bulk density (Figure

200 1b), pore volume (Figure 1c) and average pore size (Figure 1d). From the ANOVA

201 analysis it can be inferred that the four variables studied are highly dependent on the

202 percentage of surfactant, while the $\mathrm{T} / \mathrm{F}$ weight ratio significantly affects only on the

203 volume and average size of the pores.

205 Figure 1. Three-dimensional surfaces representing the effect of the T/F weight ratio and

206 percentage of surfactant on the porosity (a), bulk density (b), pore volume (c) and 207 average pore size (d) of tannin-based carbon xerogels.

\subsection{Effect of the surfactant}

210 In Figures 1a and 1b, clear differences can be observed when the percentage of

211 surfactant is modified. By adding $10 \mathrm{wt}$ \% of surfactant, all the carbon xerogels exhibit

212 the lowest density and highest porosity values, $0.34 \mathrm{~g} / \mathrm{cm}^{3}$ and $78 \%$, respectively. An

213 increase in the percentage of surfactant to above $10 \mathrm{wt}$ \% causes a slight decrease in

214 porosity from $78 \%$ to $70 \%$. Conversely, a decrease to below $10 \mathrm{wt}$. $\%$ leads to materials

215 with a poorly developed porous structure, with an average porosity of less than $20 \%$ if

216 no surfactant is added.

218 The effect of the anionic surfactant (SDS) on the final porous structure of TF carbon

219 xerogels can be attributed to the polymerization of the tannin and formaldehyde. Since reactive hydroxyl groups give tannin a reactivity towards formaldehyde similar to resorcinol (Amaral-Labat et al., 2013a; Arbenz and Averous, 2014), it is possible to

222 deduce a mechanism for the reaction based on the well-known polymerization reaction 
223 between resorcinol and formaldehyde. Hence, the first step in the polymerization of 224 tannin and formaldehyde can be assume to be the formation of tannin anions via 225 hydrogen abstraction (Rey-Raap et al., 2014). Formaldehyde is then added to them to 226 form hydroxymethyl derivatives which are linked to each other by methylene and ether 227 bonds. As the reaction proceeds, the number of bonds between the rings increases, 228 leading to the formation of the polymer backbone and, in turn, three-dimensional cross229 linked polymer clusters (Rey-Raap et al., 2014). However, two points must be borne in 230 mind when using tannin as precursor for the synthesis of carbon gels (Rey-Raap et al., 231 2015): i) the larger amount of $\mathrm{OH}$ groups in tannin molecules compared to those of 232 resorcinol will probably leave a larger number of unreacted $\mathrm{OH}$ groups and ii) as the 233 large size and lack of flexibility of tannin molecules cause steric hindrance, the addition 234 of formaldehyde will be less effective than if resorcinol is used. As a result, a poorly 235 branched structure, whose mechanical strength will not be able to counteract the 236 capillary forces created during drying, is formed. Hence, the material shrinks, leading to 237 the collapse of the polymeric structure and to a poorly developed porosity. The addition 238 of sodium dodecyl sulfate (anionic surfactant) prevents shrinkage due to the repulsive 239 forces among the tannin anions generated during the polymerization reaction and, as a 240 result, the structure of the xerogel forms further away from the SDS micelles (Matos et 241 al., 2006). Furthermore, due to these repulsive forces, the formation of methylene and 242 ether bonds is retarded and, consequently, a longer time is required for the completion 243 of the sol-gel reaction. The time required for the accomplishment of the sol-gel reaction 244 is directly related to the growth and formation of clusters: longer times lead to the 245 formation of a small number of large clusters. The differences in cluster size are clearly 246 shown in Figure 2a and 2b, where SEM images of samples with 5 wt. \% and 10 wt. \% 247 of surfactant are displayed. An increase in the concentration of surfactant from $5 \mathrm{wt} . \%$ 
to $10 \mathrm{wt} . \%$ gives rise to structures with larger clusters and, as a result, with a greater porosity (Figure 1a). On the other hand, an excessive amount of surfactant leads to the formation of a poorly cross-linked polymeric structure and to a more heterogeneous material (Figure 2c) with a broader pore size distribution, as shown in Figure S2 in the Supporting Information.

Figure 2. SEM images of organic TF xerogels with 5 wt. \% (a), 10 wt. \% (b) and 20 wt. $\%$ (c) of surfactant.

The elemental analysis data in Table S5 in the supporting information clearly show that the anionic surfactant is prevalent in the organic samples, since the sulphur content increases from 0 to $7-9$ wt. \% (on dry basis) when the amount of surfactant is increased from 0 to 20 wt. \%. The infrared spectroscopy spectra of the organic xerogels in Figure 3 indicate that the surfactant interacts with the polymeric structure, as a result of which new functionalities are incorporated into the final material.

Figure 3. FT-IR spectra of SDS anionic surfactant and organic TF xerogels with 5 wt.

$$
\%, 10 \text { wt. } \% \text { and } 20 \text { wt. } \% \text { of surfactant. }
$$

267 Sample TF-1.2-0\% presents a more intense band at around $3300 \mathrm{~cm}^{-1}$, associated to O-H 268 stretching vibrations, than samples TF-1.2-10\% and TF-1.2-20\%, and a more intense

269 band at $c a .1600 \mathrm{~cm}^{-1}$ attributed to the characteristic modes of aromatic rings (Lochab et al., 2014). This is probably due to the incorporation of the carbonaceous chains of the surfactant that gives rise to materials with a lower concentration of $\mathrm{OH}$ groups and to a

272 lower aromatic ring content. On the other hand, the incorporation of SDS induces a 
273 higher concentration of aliphatic $\mathrm{C}-\mathrm{H}$, as a result of which samples TF-1.2-10\% and TF-

$2741.2-20 \%$ display more intense bands at $2900-2800 \mathrm{~cm}^{-1}$ and $1400-1500 \mathrm{~cm}^{-1}$ than the

275 pristine organic xerogel (Viana et al., 2012). Also, samples with surfactant present a

276 new type of surface group corresponding to the sulphur content and as a consequence,

277 an absorption band at $c a .1230 \mathrm{~cm}^{-1}$ attributed to the $\mathrm{SO}_{2}$ asymmetric vibrations appears

278 (Viana et al., 2012). This functional group is probably also responsible for the presence

279 of the peaks at wavenumbers lower than $1100 \mathrm{~cm}^{-1}$. Some authors point out that the

280 values corresponding to these peaks may vary according to the way in which the

281 molecules are packed (Viana et al., 2012).

282

283 The data obtained from the infrared spectroscopy spectra are in good agreement with

284 the results of the elemental analysis of the carbon xerogels in Figure 4. The results

285 obtained are in agreement with the idea that not all the surfactant is released as volatile

286 matter during carbonization, probably due to the strong affinity between sulphur and

287 carbon. These results are of great interest, as not only are cheaper and more

288 environmentally-friendly materials obtained, but also S-doped materials, which are

289 useful for a wide range of applications (Kiciński et al., 2014).

290

291 Figure 4. Sulphur content in organic and carbon xerogels.

292

2933.3 Effect of formaldehyde

294 The T/F weight ratio hardly affects the density and porosity of the carbon xerogels

295 within the range studied (Figure $1 \mathrm{a}$ and $1 \mathrm{~b}$ ). However, the volume and average size of

296 the pores differ greatly when the T/F weight ratio is modified. In general, as with RF

297 carbon xerogels, an increase in the concentration of formaldehyde shifts the chemical 
equilibrium towards the products, favouring the addition reaction. This suggests that

299 clusters form and grow faster, resulting in TF structures with a large number of small

300 clusters, as illustrated in Figure 5. However, in TF carbon xerogels, there is also

301 interdependence between the effect of the formaldehyde and that of the surfactant, as

302 shown by the data from the statistical analysis mentioned above, on the basis of which

303 different porous structures can be tailored depending on the SDS-T/F combination

304 employed to prepare the precursor solution.

Figure 5. SEM images of carbon xerogels with $10 \mathrm{wt}$ \% of SDS and a T/F weight ratio

$$
\text { of } 2.6(\mathrm{a}) \text { and } 1.2(\mathrm{~b}) \text {. }
$$

309 Formaldehyde not only accelerates the formation and growth of clusters but also creates

310 more interconnections between the clusters (Rey-Raap et al., 2014), leading to highly

311 branched structures. Hence, when the concentration of surfactant is low (below $10 \mathrm{wt}$.

$312 \%)$, carbon xerogels synthesized with a large amount of formaldehyde have a lower

313 volume of pores of smaller size, as shown in Figure 6. As can be seen, the pore sizes

314 range from 0.8 to $11 \mu \mathrm{m}$.

316 Figure 6. Pore volume (columns) and average pore size (dots) of carbon xerogels 317 synthesized from precursor solutions with different $\mathrm{T} / \mathrm{F}$ weight ratios and surfactant 318 concentrations.

320 Due to the synergy between the formaldehyde and surfactant, the effect of 321 formaldehyde can be reversed by increasing the concentration of surfactant to above 10 322 wt. \%. This is probably because the higher number of micelles slows down the reaction 
rate, causing the formation of a smaller number of clusters but of larger size, and also

324 because of the more heterogeneous material (Figure 2). This effect, combined with the fact that a smaller concentration of formaldehyde gives rise to weaker structures, results in materials with a mechanical strength that is not able to counteract the capillary forces created during drying and hence the materials shrink. As a result, materials with a lower volume of pores of smaller average size are obtained (Figure 6).

The samples with 10 wt. \% of surfactant deserve special mention because in their case,

331 an increase in the $\mathrm{T} / \mathrm{F}$ weight ratio results in a large increase in average pore size,

332 whereas the pore volume decreases slightly, as shown in Figure 6. This behaviour is

333 opposite to that generally found for carbon xerogels where structures with smaller pores

334 also have lower pore volumes (Rey-Raap et al., 2014). In the present study, an increase in the $\mathrm{T} / \mathrm{F}$ weight ratio leads to the formation of a small number of large clusters (Figure 5) and therefore to pores of a larger size (Figure 6). However, as the concentration of

337 formaldehyde is lower, the structure is less branched and a certain degree of shrinkage 338 takes place during drying, resulting in a slight decrease in pore volume.

340 In general, when the amount of surfactant increases, porosity passes through a

341 maximum, while when the $\mathrm{T} / \mathrm{F}$ weight ratio is modified, the porosity increases or

342 decreases depending on the amount of surfactant added, due to the high interdependence

343 between these two variables. From these results, it can be seen that the right choice of

344 SDS-T/F combination is essential for controlling the formation of the polymeric structure and for tailoring the porous properties of tannin-based carbon xerogels so that

346 they fit the requirements of a specific application. 
348 As an example to demonstrate the importance of being able to control the porous

349 properties, the thermal conductivity of the organic TF xerogels synthesized by adding

35010 wt. $\%$ of surfactant and different concentrations of formaldehyde (TF-2.6-10\%, TF-

$351 \quad 1.2-10 \%$ and TF-0.6-10\%) was measured, taking into account that materials are

352 considered insulating if their thermal conductivity value is typically lower than 50

$353 \mathrm{~mW} / \mathrm{m} / \mathrm{K}$. As mentioned above, Figure 6 shows that as the amount of formaldehyde

354 increases, the average pore size decreases, whereas the pore volume increases. These

355 porous properties have a great effect on the thermal conductivity of the materials.

356 Sample TF-2.6-10\% exhibits a thermal conductivity of $65 \mathrm{~mW} / \mathrm{m} / \mathrm{K}$, corresponding to a

357 poor insulating material, whilst sample TF-1.2-10\% and TF-0.6-10\% present values of

35851 and $39 \mathrm{~mW} / \mathrm{mK}$, respectively. In short, modification of the formaldehyde

359 concentration gives rise to materials with porous properties that lead to different

360 insulation capacities. Accordingly, in order to use carbon xerogels as insulating

361 materials the appropriate process conditions should be chosen.

362

3634 Conclusions

364 In the present work, highly porous tannin-based carbon xerogels have been successfully

365 synthesized. An appropriate selection of the amount of surfactant and formaldehyde

366 allows controlling the formation of the polymeric structure and thus, the porous

367 properties. Materials synthesized are more cost-effective and environmentally "greener"

368 than the commonly used resorcinol-formaldehyde xerogels, due to the use of tannin, a

369 natural compound industrially obtained from wood. Moreover, tannin-based xerogels

370 can be compared to resorcinol-formaldehyde xerogels with respect to porosity and

371 density. However, these "greener" materials have higher volume of large pores due to

372 the nature of tannin molecules and the need to use surfactants. Hence, tannin-based 
carbon xerogels synthesized have certain limitations for those applications requiring narrow mesopores, but are totally useful in those applications in which larger pore sizes are required. Furthermore, an additional advantage is that the surfactant used in the present work allows sulphur to remain trapped inside the structure, which can be used as

377 a basis for obtaining S-doped carbons.

\section{Acknowledgements}

380 Financial support from the Ministerio de Economía y Competitividad of Spain MINECO (under Projects MAT2011-23733, IPT-2012-0689-420000 and CTQ201349433-EXP) is greatly acknowledged. NRR is also grateful to MINECO for her

383 predoctoral research grant. The French authors also gratefully acknowledge the

384 financial support of the CPER 2007-2013 ' Structuration du Po^ le de Compe' titivite'

385 Fibres Grand'Est' (Competitiveness Fibre Cluster), through local (Conseil Général des

386 Vosges), regional (Région Lorraine), national (DRRT and FNADT) and European 387 (FEDER) funds.

\section{References}

390 Al-Muhtaseb, S.A., Ritter, J.A., 2003. Preparation and properties of resorcinol-

391 formaldehyde organic and carbon gels. Adv. Mater. 15, 101-114.

392 Amaral-Labat, G., Grishechko, L.I., Fierro, V., Kuznetsov, B.N., Pizzi, A., Celzard, A., 393 2013a. Tannin-based xerogels with distinctive porous structures. Biomass Bioenerg. 56, $394 \quad 437-445$.

395 Amaral-Labat, G., Szczurek, A., Fierro, V., Celzard, A., 2015. Unique bimodal carbon 396 xerogels from soft templating of tannin. Mater. Chem. Phys. 149-150, 193-201. 
397 Amaral-Labat, G., Szczurek, A., Fierro, V., Pizzi, A., Celzard, A., 2013b. Systematic

398 studies of tannin-formaldehyde aerogels: preparation and properties. Science and

399 Technology of Advanced Materials 14, 015001.

400 Arbenz, A., Averous, L., 2014. Synthesis and characterization of fully biobased 401 aromatic polyols - oxybutylation of condensed tannins towards new macromolecular 402 architectures. RSC Adv. 4, 61564-61572.

403 Braghiroli, F.L., Fierro, V., Izquierdo, M.T., Parmentier, J., Pizzi, A., Celzard, A., 2014. 404 Kinetics of the hydrothermal treatment of tannin for producing carbonaceous 405 microspheres. Bioresour. Technol. 151, 271-277.

406 Braghiroli, F.L., Fierro, V., Izquierdo, M.T., Parmentier, J., Pizzi, A., Delmotte, L., 407 Fioux, P., Celzard, A., 2015a. High surface - Highly N-doped carbons from 408 hydrothermally treated tannin. Ind. Crops Prod. 66, 282-290.

409 Braghiroli, F.L., Fierro, V., Szczurek, A., Stein, N., Parmentier, J., Celzard, A., $2015 b$.

410 Electrochemical performances of hydrothermal tannin-based carbons doped with 411 nitrogen. Ind. Crops Prod. 70, 332-340.

412 Elkhatat, A.M., Al-Muhtaseb, S.A., 2011. Advances in tailoring resorcinol413 formaldehyde organic and carbon gels. Adv. Mater. 23, 2887-2903.

414 García, D.E., Glasser, W.G., Pizzi, A., Lacoste, C., Laborie, M.P., 2014. Polyphenolic 415 resins prepared with maritime pine bark tannin and bulky-aldehydes. Ind. Crops Prod. $416 \quad 62,84-93$.

417 Job, N., Heinrichs, B., Ferauche, F., Noville, F., Marien, J., Pirard, J.-P., 2005.

418 Hydrodechlorination of 1,2-dichloroethane on $\mathrm{Pd}-\mathrm{Ag}$ catalysts supported on tailored 419 texture carbon xerogels. Catal. Today 102-103, 234-241.

420 Kiciński, W., Szala, M., Bystrzejewski, M., 2014. Sulfur-doped porous carbons: 421 Synthesis and applications. Carbon 68, 1-32. 
422 Lochab, B., Shukla, S., Varma, I.K., 2014. Naturally occurring phenolic sources:

423 monomers and polymers. RSC Adv. 4, 21712-21752.

424 Matos, I., Fernandes, S., Guerreiro, L., Barata, S., Ramos, A.M., Vital, J., Fonseca, 425 I.M., 2006. The effect of surfactants on the porosity of carbon xerogels. Micropor. 426 Mesopor. Mater. 92, 38-46.

427 Rey-Raap, N., Menéndez, J.A., Arenillas, A., 2014. Simultaneous adjustment of the 428 main chemical variables to fine-tune the porosity of carbon xerogels. Carbon $78,490-$ 429499.

430 Rey-Raap, N., Szczurek, A., Fierro, V., Menéndez, J.A., Arenillas, A., Celzard, A., 431 2015. Towards a feasible and scalable production of bio-xerogels. J. Colloid Interface 432 Sci. 456, 138-144.

433 Roberts, A.D., Li, X., Zhang, H., 2014. Porous carbon spheres and monoliths: 434 morphology control, pore size tuning and their applications as Li-ion battery anode 435 materials. Chem. Soc. Rev. 43, 4341-4356.

436 Szczurek, A., Amaral-Labat, G., Fierro, V., Pizzi, A., Celzard, A., 2011a. The use of 437 tannin to prepare carbon gels. Part II. Carbon cryogels. Carbon 49, 2785-2794.

438 Szczurek, A., Amaral-Labat, G., Fierro, V., Pizzi, A., Masson, E., Celzard, A., 2011 b. 439 The use of tannin to prepare carbon gels. Part I: Carbon aerogels. Carbon 49, 27734402784.

441 Szczurek, A., Fierro, V., Pizzi, A., Stauber, M., Celzard, A., 2014. A new method for 442 preparing tannin-based foams. Ind. Crops Prod. 54, 40-53.

443 Tannert, R., Schwan, M., Ratke, L., 2015. Reduction of shrinkage and brittleness for 444 resorcinol-formaldehyde aerogels by means of a pH-controlled sol-gel process. J. 445 Supercrit. Fluids 106, 57-61. 
446 Viana, R.B., da Silva, A.B.F., Pimentel, A.S., 2012. Infrared Spectroscopy of Anionic,

447 Cationic, and Zwitterionic Surfactants. Advances in Physical Chemistry 2012, 14.

448 Yang, T., Liu, J., Zhou, R., Chen, Z., Xu, H., Qiao, S.Z., Monteiro, M.J., 2014. N-doped

449 mesoporous carbon spheres as the oxygen reduction reaction catalysts. Journal of 450 Materials Chemistry A 2, 18139-18146.

451 Zubizarreta, L., Arenillas, A., Pis, J.J., 2009. Carbon materials for H2 storage. Int. J. 452 Hydrogen Energy 34, 4575-4581.

453 